\title{
Knowledge and practice of standard precautions in public health facilities in Abuja, Nigeria
}

\author{
Emeka Franklin Okechukwu', Chauke Motshedisi² \\ 1 U.S. Agency for International Development, Nigeria \\ ${ }^{2}$ Department of Health Studies, University of South Africa, South Africa
}

\begin{abstract}
Health Care Workers (HCWs) are at risk of exposure to infections during the course of carrying out their duties. The practice of standard precautions among healthcare workers (HCWs) in public health facilities in Abuja, Nigeria was evaluated. A cross-sectional survey of $277 \mathrm{HCWs}$ was conducted using a structured questionnaire.

Of 277 participants, 83 (29.96\%) were doctors; 194 (70.04\%) were nurses. Median age (in years) of doctors was 34 (IQR: 30, 39), while nurses was 39 (IQR: 33, 45). Median years of experience for doctors was 7 (IQR: 4,10 ) while nurses was 15 (IQR: 9, 20). Only $16.61 \%$ of participants had knowledge of the basic concept of standard precautions and $42.24 \%$ knew potential sources of occupational exposure. The association between the knowledge of the potential sources of occupational exposures and profession was significant $(\mathrm{p}=0.011)$. Hand washing was practiced by $97.46 \% ; 97.83 \%$ reported regular use of hand gloves; $88.44 \%$ use gown or plastic apron; $68.95 \%$ use masks and eye protector. The major factor reported for non-adherence was nonavailability of the materials. Practice of recapping used needle was more common among doctors than nurses $(p=0.01$ ). The HCWs hepatitis-B vaccination status was poor; $32.53 \%$ of doctors and $28.87 \%$ of nurses had $\geq 1$ dose of hepatitis-B vaccine. The difference in hepatitis-B immunization status of doctors and nurses was not significant $(\mathrm{p}=0.556)$.
\end{abstract}

\section{Key words}

Occupational exposure, prevention and control; health personnel; cross-sectional studies;

infection control, standards

\section{Corresponding Author}

Emeka Franklin Okechukwu

Treatment Manager, HIV/AIDS \& TB, U.S. Agency for International Development, Nigeria

Email: drlongusf@yahoo.co.uk 
The knowledge of standard precautions among HCWs was suboptimal. Except for mask and eye protector, the use of PPE was good. Non-availability of materials was the major cause of poor compliance. Capacity development of HCWs and a comprehensive infection prevention and control program were recommended.

\section{Introduction}

Health Care Workers (HCWs) are potentially exposed to infections while performing their duties. Standard precautions is regarded as an effective means of protecting HCWs, patients, and the public, thus reducing hospital acquired infections. ${ }^{1}$ Standard precautions are designed to protect health care workers from being exposed to potentially infected blood and body fluid by applying the fundamental principles of infection prevention, through hand washing, utilization of appropriate protective barriers such as gloves, mask, gown, and eye wear. ${ }^{2}$ Standard precautions are also intended to protect the patient by ensuring that healthcare personnel do not transmit infectious agents to patients through their hands or equipment during patient care. ${ }^{3}$

The practice of standard precautions is being widely promoted to protect HCWs from occupational exposure to body fluids and consequent risk of infection with blood-borne pathogens; however, the situation may be different in low-income countries. ${ }^{4}$ HCWs frequently provide care to patients who may be asymptomatic while being infectious. WHO states that worldwide, about $40 \%$ of Hepatitis B and C Virus infections and $2.5 \%$ of HIV infections to HCWs are attributable to occupational sharps exposures, which are mainly preventable. ${ }^{5}$ The risk of acquiring HBV infection from occupational exposure depends on the frequency of percutaneous and per mucosal exposures to blood or body fluids containing blood. ${ }^{6}$ Although percutaneous injuries are among the most efficient modes of HBV transmission, percutaneous exposures probably account for only a minority of HBV infections among HCWs. In several investigations of nosocomial hepatitis B outbreaks, most infected HCWs could not recall an overt percutaneous injury. ${ }^{7}$ However, in some studies, up to one-third of infected HCWs recalled caring for a patient who was HBsAg-positive. ${ }^{8}$ In addition, HBV has been demonstrated to survive in dried blood at room temperature on environmental surface for at least one week. ${ }^{9}$ Thus, HBV infections that occur in HCWs with no history of occupational exposure or occupational percutaneous injury might have resulted from direct or indirect blood or body fluid exposures that inoculated HBV into cutaneous scratches, abrasions, burns, other lesions, or mucosal surfaces. ${ }^{10}$ Because of the high risk of HBV infection among HCWs, routine pre-exposure vaccination of $\mathrm{HCW}$ s against hepatitis $B$ and the use of standard precautions to prevent exposure to blood and other potentially infectious body fluids have been recommended since 1980s. ${ }^{11}$

Compliance to standard precautions is low in public health facilities, especially in resource-limited settings such as Nigeria, thus exposing HCWs to the risk of infection. Sagoe-Moses et al. ${ }^{12}$ noted that occupational safety of HCWs is often neglected in low-income countries in spite of the greater risk of infection due to higher disease prevalence, inadequate supply of personal protective equipment (PPE) and limited organizational support for safe practices. Needle stick injuries have been shown to be the commonest (75.6\%) mechanism for occupational exposure in a Nigerian teaching hospital. ${ }^{13}$ These injuries are usually under-reported for so many reasons, which include stigma that could be associated with an eventual infection with HIV in the affected HCW. There is no immunization for HIV and HCV, thus the most effective prevention is through regular practice of the standard precautions.

The purpose of this study is to determine the knowledge, practice and factors affecting the utilization of standard precautions among Health Care Workers (HCWs) in public secondary health facilities in Abuja, Nigeria.

\section{Methods}

A quantitative descriptive cross-sectional survey was conducted among two health care worker professions i.e. doctors and nurses in public secondary healthcare facilities in Abuja, Nigeria. Multistage sampling technique was used for sample selection. A list of public secondary health facilities in Abuja was obtained from the Health and Human Services Secretariat (HHSS) of the Federal Capital Territory Authority (FCTA) Abuja; eight secondary health facilities were selected randomly out of the 10 public secondary health facilities in Abuja. Selection of study participants 
was done in the second stage. In each of the selected hospitals, a list of names of the doctors and nurses was obtained from the hospital administrative authority. Simple random sampling technique was applied to obtain eligible respondents. Where a selected individual was unavailable or declined to participate in the study, the next personnel on the list was chosen.

The sample size (277) was calculated using the formula ${ }^{14}$

$$
\mathrm{nf}=\frac{\mathrm{n}}{1+\underline{(\mathrm{n})}}(\text { for population }<10,000)
$$

$(\mathrm{N})$

\section{Where:}

$\mathrm{nf}=$ the desired sample size when the population is less than 10,000

$\mathrm{n}=384$, i.e. the desired sample size when the population is more than 10,000 (Calculated using Cochrane formula ${ }^{15}$ )

$N=1000$, i.e. the estimate of the population size (doctors and nurses in Abuja) provided by FCTA).

An anonymous self-administered pre-coded structured questionnaire was used to assess the knowledge and practice of standard precautions among these two professions of HCWs. The questionnaire has three main sections; the first part comprised 4 questions on biographical data (age and gender), profession and post-qualification experience. The second part contained 9 questions which sought to ascertain the level of knowledge and understanding of the concept of standard precautions including post-exposure prophylaxis. The third part comprised 20 questions on the level of adherence to standard precautions. Included also in this section were questions on the availability and use of guidelines for Post Exposure Prophylaxis (PEP). The data collection tool was pretested on HCWs from hospitals not included in the study. None of those involved in the pre-test participated in the actual study. Each participant in the pre-test was requested to critically analyse all aspects of the questionnaire and to comment on the relevance of the questions, wording and clarity of questions, length of the questionnaire and the time required to complete the questionnaire as well as inadequate or confusing response categories. Findings from the pretest exercise were used to revise the questionnaire.
Data analysis was done using STATA version 10 statistical software. Descriptive statistics were used in the univariate analysis, which includes frequency distribution of key items. Bivariate analysis (cross tabulation) was used to describe the study participants and the knowledge and practice of standard precautions among the respondents.

\section{Results}

A total of 277 health care workers participated in this study. Of these, $83(29.96 \%)$ were doctors and 194 $(70.04 \%)$ were nurses. Respondents' aged $30-39$ years had the highest frequency $(45.85 \%)$ and those aged 50-55 years had the lowest frequency $(4.69 \%)$. The median age of doctors was 34 (IQR: 30, 39) and for nurses 39 (IQR: 33, 45) years. The oldest doctor among the respondents was 52 years while the oldest nurse was 55 years (Figure 1). Thirty-four (40.96\%) doctors had less than five years post-graduation professional experience and 94 (48.45\%) of the nurses had more than 15 years of post-graduation professional experience. Thirty-one (37.35\%) doctors and 44 (22.68\%) nurses had 6-10 years professional experience. The median years of professional experience for doctors was 7 (IQR: 4, 10) and for nurses 15 (IQR: 9, 20). The maximum years of professional experience for doctors and nurses were 22 and 30 respectively.

\section{Knowledge of Standard Precautions:}

Approximately $17 \%$ of the respondents answered correctly and completely on the basic concept of standard precautions which includes hand washing before and after any direct contact with patient, consideration of the potential for transmission of infectious agents in patient placement decisions, cough etiquette such as directing patients/relatives with symptoms of a respiratory infection to cover their mouths/noses when coughing or sneezing and safe injection practices such as use aseptic technique (Table I). At $5 \%$ level of significance, there was no association between the knowledge of the basic concept of standard precautions and profession $(\mathrm{p}=$ 0.283). Less than half of the respondents $(42.24 \%)$ answered correctly and completely the question on the potential sources of occupational exposure which includes needle stick /sharp injury, splash on the eye, inhalation and touching patients. There was statistical significant relationship between the knowledge of 
Table I. Frequency distribution of HCWs who knew the standard precautions

\begin{tabular}{lccr} 
Knowledge of standard precautions & Doctors (\%) & Nurses (\%) & Total (\%) \\
\hline Basic concept of standard precautions & $23(27.71)$ & $23(11.86)$ & $46(16.61)$ \\
Potential sources of occupational exposure & $44(53.01)$ & $73(37.63)$ & $117(42.24)$ \\
Situations requiring hand washing & $20(24.10)$ & $42(21.65)$ & $62(22.38)$
\end{tabular}

the potential sources of occupational exposure and profession $(p=0.011)$.

The majority of respondents $(90.36 \%$ of doctors and $91.75 \%$ of nurses) were aware that standard precautions should apply to all patients irrespective of their diagnosis. Knowledge of the recommendation on post-exposure prophylaxis (PEP) was poor among the respondents. About half of the respondents $(49 \%$ of doctors and $56.7 \%$ nurses) knew that HIV counselling and testing was required after occupational exposure, and that PEP should be given if the HIV test result of the exposed person is negative; in accordance with the National guideline by Federal Ministry of Health (FMOH) ${ }^{16}$ Higher proportion of respondents $(85.54 \%$ of doctors and $69.07 \%$ of nurses) knew that two or three antiretroviral drugs should be used for PEP within 72 hours of exposure (Table II). Also $68.67 \%$ of doctors and $62 \%$ of nurses knew the correct duration for PEP. Low proportion of the respondents (32.53\% of doctors and $27.84 \%$ of nurses) was aware of the availability of the HIV PEP guideline in their facility while $15.52 \%$ of the respondents were aware of the presence of a PEP focal person in their facility.

\section{Practice of standard precautions:}

The majority of the HCWs $196.38 \%$ of doctors and $97.94 \%$ of nurses) reported that they practiced hand washing with soap and water after any direct contact with patients (Table III). However, a lower proportion ( $60 \%$ of doctors and $72 \%$ of nurses) reported always practicing hand washing while $3.51 \%$ of doctors and $2.06 \%$ of nurses reported that they seldom washed their hands. There was no report of doctors and nurses who never washed hands. There was no statistical significant relationship $(p=0.118)$ between hand washing by doctors and nurses, but there was association between hand washing and years of professional experience $(p=0.002)$ (Table III). High proportion of the respondents $(97.83 \%)$ reported regular use of gloves when contact with body fluids, non-intact skin and mucous membrane is anticipated. There was statistical significant relationship between the use of gloves and profession. The major constraint

Table II. Knowledge of post-exposure prophylaxis

\section{Knowledge of PEP}

\section{Doctors \\ Nurses}

$\begin{array}{llll}\text { Yes }(\%) & \text { No }(\%) & \text { Yes }(\%) & \text { No }(\%)\end{array}$

HIV Counselling \& Testing required immediately after exposure and PEP given to HIV negative.

$42(50.60) \quad 110(56.70)$

$84(43.30)$

2 or 3 Antiretroviral drugs are used for PEP within 72 hours of exposure.

$12(14.46) \quad 134(69.07)$

$60(30.93)$

PEP should be taken for 4 weeks
$57(68.67)$ $26(31.33) \quad 121(62.37)$ 


\section{Table III. Frequency distribution of hand washing by respondents}

\begin{tabular}{lrrr} 
Hand washing & Doctors & Nurses & Total (\%) \\
\hline Always & $\mathbf{N}(\%)$ & $\mathbf{N}(\%)$ & $191(68.95)$ \\
Often & $50(60.24)$ & $141(72.68)$ & $79(28.51)$ \\
Seldom & $30(36.14)$ & $49(25.26)$ & $7(2.52)$ \\
Never & $3(3.61)$ & $4(2.06)$ & 0 \\
\hline Total & 0 & 0 & $\mathbf{2 7 7}$
\end{tabular}

reported by the few respondents who fail to adhere to the basic principles of hand washing and the use of glove was that water, soap and gloves were not readily available.

The majority of the respondents $(88.44 \%)$ reported that they wore gown or plastic apron during procedures likely to generate splashes of blood or body fluid. Few respondents $(7.94 \%)$ who seldom used gown or plastic apron during procedures reported lack of supplies as the reason for irregular or non-use of gown or plastic apron. More than half of the respondents $(68.95 \%)$ reported using mask and eye protector for procedures likely to generate droplets or splash of blood or body fluid. Again, non-availability of masks and eye protector was reported as the reason for irregular use of these personal protective equipments by the respondents.

Practice of recapping used needle is commoner among doctors $(24.10 \%)$ than nurses $(11.86 \%)$. There was association between profession and recapping of needle after use $(p=0.01)$. Nineteen $(9.79 \%)$ nurses and $14(16.87 \%)$ doctors reported needle stick injury in the last three months. It is not surprising to see more doctors reporting needle stick injury, since more doctors than nurses reported recapping needle after use. There was no statistical significant relationship ( $p$ $=0.096$ ) between the incidence of needle stick injury and profession (doctors and nurses). However, the incidence of needle stick injuries is an indirect marker for measuring the level of adherence to standard precautions by HCWs. High proportion of doctors $(97.59 \%)$ and nurses $(89.19 \%)$ reported that sharp disposal containers were located as close as possible to the use area.

A low proportion of the respondents $32.53 \%$ of doctors and $28.87 \%$ of nurses) had at least one dose of hepatitis B vaccine. Among those that received hepatitis B vaccine, a very low proportion $(18.51 \%$ of doctors and $39.28 \%$ of nurses) had the complete 3 doses. There was no statistical significant relationship between profession and hepatitis B immunization status $(p=0.556)$.

\section{Discussion}

\section{Knowledge of standard precautions among health care workers:}

Knowledge of the basic concept of the standard precautions was inadequate among the health care workers; few respondents answered correctly all questions on the components of the concept of standard precautions. A similar study to assess the knowledge and compliance with universal precautions and their perceived risk of infection at the workplace conducted in Ibadan, Nigeria revealed that some $77.5 \%$ of the respondents were aware but only $24 \%$ had the correct knowledge of the universal precautions. ${ }^{17}$ They had inadequate knowledge of the potentials sources for transmission of infectious agents and situations requiring hand washing before and after any direct contact with patients. In contrast, the majority of HCWs knew that standard precautions should apply to all patients and to all body fluids irrespective of the diagnosis. HCWs were more familiar with this aspect of the standard precautions. There was statistical significant relationship between the knowledge of the 
Table IV. Respondents' Hepatitis B vaccination status

\begin{tabular}{lrrrr} 
& \multicolumn{2}{c}{ Doctors } & \multicolumn{2}{c}{ Nurses } \\
\cline { 2 - 5 } & Yes $(\%)$ & No $(\%)$ & Yes (\%) & No (\%) \\
\hline Had hepatitis B vaccination & $27(32.53)$ & $56(67.47)$ & $56(28.87)$ & $136(70.10)$
\end{tabular}

potential ways of occupational exposure among the doctors and nurses $(p=0.011)$.

There was variation in the knowledge of post-exposure prophylaxis among the HCWs. The knowledge of the critical step of conducting HIV counselling and testing immediately after exposure and PEP given only to the exposed person that test negative was low among the respondents. A higher proportion of the respondents knew that two or three antiretroviral drugs should be used within 72 hours of exposure and should be taken for duration of four weeks. The low proportion of respondents who were aware of the PEP focal person is an indication of poor occupational accident reporting and documentation; thus the need to establish appropriate post-exposure management system that includes written protocols for prompt reporting, evaluation, counselling, and treatment as well as follow-up of occupational exposures.

\section{Practice of Standard Precautions among Health Care Workers:}

A good proportion of the HCWs reported irregular washing of hands with soap and water after any direct contact with patients, which is a simple routine infection prevention measure. The major constraint reported by respondents who said they did not always wash hands with soap and water after any direct contact with patients was irregular availability of water and soap.

Findings from the study show that the majority of the respondents always use gloves when they anticipate contact with body fluids, non-intact skin and mucous membrane. However, the use of other personal protective equipment such as gown or plastic apron, mask and eye protector by respondents during procedures likely to generate droplets/splashes of blood or body fluid was low. The main constraint given for the irregular use of PPE was lack of regular supplies.
Recapping of needle after use was low among the respondents in general but higher among doctors than nurses. This result is similar to that of a related study conducted among health care workers in public and private health care facilities in Abeokuta metropolis in Nigeria which showed that about one-third of all respondents always recapped used needles and that the practice of recapping of needles was highest among doctors but less among trained nurses. ${ }^{18} \mathrm{~A}$ higher proportion of respondents disposed used needles in puncture-proof containers and the majority of them reported that sharps disposal containers were located as close as possible to the use area. The incidence of needle stick injury among HCWs was generally low, but it was higher among doctors than nurses. A low proportion of the respondents had at least one dose of hepatitis B vaccine, and among these only very few had the full course of the vaccine.

\section{Conclusion}

This study revealed valuable information about the knowledge and practice of standard precautions among HCWs in secondary health facilities in Abuja, Nigeria. Knowledge and practice of standard precautions, was suboptimal among HCWs. Although, the knowledge

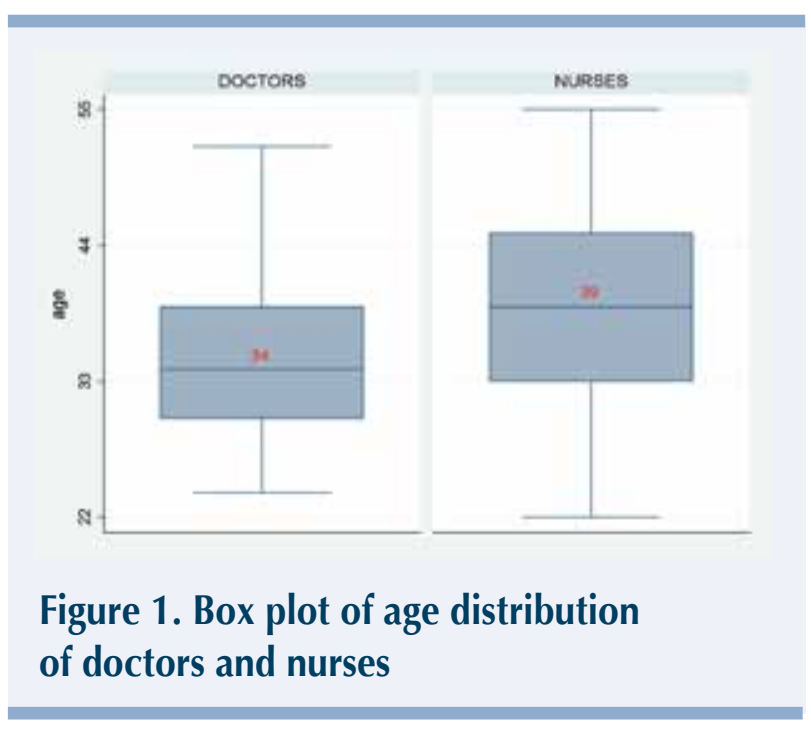


that standard precautions should be observed while dealing with all patients and body fluids irrespective of the diagnosis was high among the respondents; this knowledge however, did not seem to translate to high rate of adherence to the requirements for the use of personal protective equipment. Irregular supply of PPE and other required materials came out as one of the major cause of poor adherence to standard precautions. These findings will be useful in planning appropriate measures to improve the knowledge and compliance of HCWs to standard precautions, thus reducing the risk of occupational exposures and subsequent infection transmission among HCWs.

\section{Acknowledgements}

The authors would like to thank Titilope Badru, for analysing the data.

\section{Declaration}

An abstract of this study was published as poster presentation at the 3 rd Infection Prevention and Control Africa Network (IPCAN) Conference, Windhoek, Namibia. November 2011.

\section{References}

1. Wang $\mathrm{H}$, Fennie $\mathrm{K}$, Burgess L, Williams AB. A training programme for the prevention of occupational exposure to blood borne pathogens: Impact on knowledge, behavior and incidence of needle stick injuries among student nurses in Changsha, People's Republic of China. Journal of Advanced nursing 2003; 41(2): 187-1194. http://dx.doi.org/10.1046/ j.1365-2648.2003.02519.x

2. Motamed N, BabaMahmoodi F, Khalilian A, Peykanheirati M, Nozari M. Knowledge and practices of health care workers and medical students towards universal precautions in Mazandaran province. Eastern Mediterranean Health Journal 2006; 12(5): 1205.

3. Siegel JD, Rhinehart $E$, Jackson $M$, Chiarelo $L$ and the Health Infection Control Practices Advisory Committee. 2007. Guideline for Isolation precaution: Preventing Transmission of Infectious Agents in Healthcare Setting, 2007. Public Health Services, US Department of Health and Human Services Centre for Disease Control and Prevention Atlanta Georgia. http://www.cdc.gov/ncidod/dhqp/pdf/isolation2007.pdf [accessed 4 May 2009]
4. Kermode M, Jolley D, Langkham B, Thomas MS, Holmes W, Gifford SM. Compliance with universal/standard precautions among health care workers in rural north India. American Journal of Infection Control 2005; 33(1): 27-33. http://dx.doi. org/10.1016/j.ajic.2004.07.014

5. WHO: Reducing Risk, Promoting Health Life. World Health Report. Geneva; 2002.

6. Thomas DL, Factor SH, Gabon D, et al. Viral hepatitis in health care personnel at Johns Hopkins hospital. Arch intern Med 1993; 153: 1705-1712. http://dx.doi.org/10.1001/ archinte.1993.00410140095011

7. Garibaldi RA, Hatch FE, Bisno AL, Hatch MH, Greg MB. Non parenteral serum hepatitis: report of an outbreak. JAMA 1972; 220: 963-966. http://dx.doi.org/10.1001/ jama.1972.03200070055008

8. Callender ME, White YS, Williams R. Hepatitis B viral infection in medical and health care personnel. Br Med / 1982; 284: 324-326. http://dx.doi.org/10.1136/bmj.284.6312.324

9. Shepard CW, Simard EP, Finelli L, Fiore AE, Bell BP. Hepatitis B Virus Infection: Epidemiology and Vaccination. Epidemiol Rev 2006; 28: 114. http://dx.doi.org/10.1093/epirev/mxj009

10. Francis DP, Favero MS, Maynard JE. Transmission of hepatitis B virus. Semin Liver Dis 1981; 1: 27-32. http://dx.doi. org/10.1055/s-2008-1063927

11. CDC. Recommendation of immunization practice advisory committee; inactivated hepatitis $\mathrm{B}$ virus vaccine. MMWR 1982; 31: 317-328.

12. Sagoe-Moses C, Pearson RD, Perry J, Jagger J. Risks to health care workers in developing countries. N Engl / Med 2001; 345: 538- 541. http://dx.doi.org/10.1056/NEJM200108163450711

13. Orji EO, Fasubaa OB, Onwudiegwu U. Occupational health hazards among health care workers in an obstetrics and gynaecological unit of a Nigerian teaching hospital. Journal of Obstetrics and Gynaecology 2002; 22: 75-78. http://dx.doi. org/10.1080/01443610120101781

14. Araoye MO. 2004. Research methodology with statistics for health and social sciences. Ilorin: Nathadex.

15. Gill J, Johnson P. 2010. Research methods for managers. 4th ed., Sage, London.

16. FMOH: National guideline for HIV and AIDS treatment and care in adolescents and adult. Abuja; 2007.

17. Kolude OO, Omokhodion FO, Owoaje ET. Universal Precaution: Knowledge, Compliance and perceived risk of infection among doctors at a University Teaching Hospital. Int conf AIDS 2004; Abstract no MoPed 3676.

18. Sadoh WE, Fawole AO, Sadoh AE, Oladimeji AO, Sotiloye OS. Practice of universal precautions among health care workers. Journal of the National Medical Association 2006; 98(5): 722 726. 